\title{
Comparison of Interference Mitigation Techniques for Next Generation DSL Systems
}

\author{
Sanda Drakulić*, Driton Statovci*, Martin Wolkerstorfer*, and Thomas Zemen ${ }^{\dagger}$ \\ *FTW Telecommunications Research Center Vienna, Donau-City-Straße 1, A-1220 Vienna, Austria \\ \{drakulic, statovci, wolkerstorfer\}@ftw.at \\ ${ }^{\dagger}$ AIT Austrian Institute of Technology, Donau-City-Straße 1, 1220 Vienna, Austria \\ \{thomas.zemen\}@ait.ac.at
}

\begin{abstract}
The next digital subscriber line (DSL) generation, called G.fast, extends the frequency band up to $212 \mathrm{MHz}$ and achieves Gigabit data-rates. Vectoring technology cancels the performance-limiting crosstalk among DSL lines on a signal level, but requires co-located line termination. Differently, dynamic spectrum management level 2 (DSM-L2) coordinates the transmit power spectra among interferers and thus, does not require all line termination units to be co-located. In this work we analyze the data-rates of G.fast under full vectoring, partial vectoring restricted to groups of users, and non-vectoring scenarios. We also propose interference alignment (IA) as an alternative method to DSM-L2 for dealing with the interference in G.fast and show that IA is superior to DSM-L2 in non-vectoring scenarios. Furthermore, we also propose time division multiple access (TDMA) for coordination of multiple vectoring groups in partial vectoring scenarios.
\end{abstract}

\section{INTRODUCTION}

The International Telecommunication Union (ITU) has recently developed a new standard for digital subscriber line (DSL) systems called G.fast [1]. It targets aggregate bit-rates of up to $1 \mathrm{Gbps}$ over a maximum loop length of 250 meters and utilizes frequencies up to $212 \mathrm{MHz}$. G.fast represents the last step towards fiber-to-the-home (FTTH), i.e., an all fiber access network and it is tailored for fiber-to-the-building (FTTB) or fiber-to-the-distribution-point (FTTD) deployments. One of the main performance limitations for G.fast is electromagnetic interference, also called crosstalk, among different lines in the same cable bundle. Dynamic spectrum management (DSM) is a key technology for mitigating and canceling crosstalk noise on DSL lines and thus improving the achievable bit-rates in DSL systems. DSM algorithms are commonly organized into three levels depending on the amount of multi-user coordination applied. The degree of coordination ranges from single-line power control (DSM-L1) [2] over multi-line powerspectrum control (DSM-L2) [3] to joint signal processing (DSM-L3 or vectoring) [4]. Vectoring is capable of achieving considerably higher bit-rates than DSM-L1 and DSM-L2 but requires signal coordination among all line termination units. There are network scenarios when signal coordination is possible only among sub-groups of lines (partial vectoring),

The Competence Center FTW Forschungszentrum Telekommunikation Wien $\mathrm{GmbH}$ is funded within the program COMET - Competence Centers for Excellent Technologies by BMVIT, BMWA, and the City of Vienna. The COMET program is managed by the FFG. i.e., each user has signal level coordination with other users in its group, but not with users in other groups. Examples of this include (a) scenarios where two (or more) groups of lines are connected to line terminals that reside on different locations; $(b)$ scenarios where line terminals reside on the same location but are operated by different service providers (subloop unbundling); and (c) scenarios where users are partitioned into separate vectoring groups in order to decrease overall vectoring complexity or system level power consumption. For these scenarios, DSM-L1 or L2 have been proposed to mitigate crosstalk between vectoring groups [5], [6].

In this paper, we investigate interference mitigation techniques for partial vectoring scenarios. At high frequencies $(\geq$ $30 \mathrm{MHz})$ and short loop lengths $(\leq 250 \mathrm{~m})$ crosstalk channel gains become of comparable strength as the direct channel gains or even stronger [7], [8]. Therefore, in this work we consider interference alignment (IA) [9] as an alternative approach to DSM-L2 for dealing with residual crosstalk in partial vectoring. IA has similarly been suggested in [10] for current DSL systems and was compared to partial vectoring for frequencies below $30 \mathrm{MHz}$. The main differences of our work are as follows. Firstly, we evaluate IA on higher frequencies (up to $212 \mathrm{MHz}$ ) where consequently the signal-to-interferencenoise ratio (SINR) is much lower than on frequencies analyzed in [10]. Secondly, we use a more appropriate IA algorithm, which achieves higher bit-rates at low SINR than the one used in [10]. Thirdly, we use a more realistic channel model in our simulations. Instead of the $99 \%$ worst-case model [11] (frequently used in DSL simulations) we use a channel model which introduces crosstalk dispersion [12] with respect to the $99 \%$ model. In this way we introduce higher frequency diversity into the channel, which is essential for assessing the performance of IA. The authors in [10] conclude that IA has the potential to provide performance gains over partial vectoring but in their work they do not consider any of the interference mitigation techniques frequently used in partial vectoring (such as DSM-L2) for comparison. Contrarily, in this paper we compare IA to DSM-L2 for partial vectoring and show that none of them perform better than time division multiple access (TDMA) in the considered G.fast compliant set-up.

The rest of the paper is organized as follows. In Section II we introduce the system model and define the notation. Next, 
in Section III we describe the working principle of partial vectoring where crosstalk noise between groups is mitigated with DSM-L2 and IA. Furthermore, in Section IV we discuss interference alignment implementation issues. In Section V we show simulation results using a network topology with colocated line termination units, i.e., scenario $(b)$. In Section VI we draw conclusions from our work.

\section{SYSTEM AND PERFORMANCE MODEL}

We consider a DSL network with a set of users indexed by $\mathcal{N}=\{1, \ldots, N\}$ and set of $\Delta f[\mathrm{~Hz}]$ spaced tones indexed by $\mathcal{K}=\{1, \ldots, K\}$. We assume that the users are divided into groups indexed by $\mathcal{G}=\{1, \ldots, G\}$, each with $N_{g}$ members where $\sum_{g=1}^{G} N_{g}=N$ and $\mathcal{N}_{g}=\left\{1, \ldots, N_{g}\right\}$. We assume that interference inside each group and among different groups is managed at the signal and transmit power level, respectively. We also assume synchronous discrete multitone (DMT) transmission with all users having the same modulation parameters. Therefore, transmission can be modeled independently on each tone $k$ as

$$
\mathbf{y}_{k}=\mathbf{H}_{k} \mathbf{x}_{k}+\mathbf{z}_{k},
$$

where $\mathbf{H}_{k}$ is the channel transfer matrix, $\quad \mathbf{x}_{k} \triangleq \quad \triangleq \quad\left[\left(\mathbf{x}_{k}^{1}\right)^{\top},\left(\mathbf{x}_{k}^{2}\right)^{\top}, \ldots,\left(\mathbf{x}_{k}^{G}\right)^{\top}\right]^{\top}$, $\mathbf{y}_{k} \triangleq\left[\left(\mathbf{y}_{k}^{1}\right)^{\top},\left(\mathbf{y}_{k}^{2}\right)^{\top}, \ldots,\left(\mathbf{y}_{k}^{G}\right)^{\top}\right]^{\top}, \quad$ and $\quad \mathbf{z}_{k} \triangleq$ $\left[\left(\mathbf{z}_{k}^{1}\right)^{\top},\left(\mathbf{z}_{k}^{2}\right)^{\top}, \ldots,\left(\mathbf{z}_{k}^{G}\right)^{\top}\right]^{\top}$ are the transmitted signal, received signal and background noise vectors, respectively, all on tone $k$. Sub-vectors $\mathbf{x}_{k}^{g}, \mathbf{y}_{k}^{g}$, and $\mathbf{z}_{k}^{g}$ are the transmitted signal, received signal and background noise vectors of group $g$. Thus, Eq. (1) can also be written as follows

$$
\left[\begin{array}{c}
\mathbf{y}_{k}^{1} \\
\vdots \\
\mathbf{y}_{k}^{G}
\end{array}\right]=\left[\begin{array}{ccc}
\mathbf{H}_{k}^{1,1} & \cdots & \mathbf{H}_{k}^{G, 1} \\
\vdots & \ddots & \vdots \\
\mathbf{H}_{k}^{1, G} & \cdots & \mathbf{H}_{k}^{G, G}
\end{array}\right]\left[\begin{array}{c}
\mathbf{x}_{k}^{1} \\
\vdots \\
\mathbf{x}_{k}^{G}
\end{array}\right]+\left[\begin{array}{c}
\mathbf{z}_{k}^{1} \\
\vdots \\
\mathbf{z}_{k}^{G}
\end{array}\right],
$$

where $\mathbf{H}_{k}^{g, g^{\prime}}, g \neq g^{\prime}$ contains crosstalk channel gains from group $g^{\prime}$ to group $g$ while $\mathbf{H}_{k}^{g, g}$ contains direct channel gains (diagonal elements) and crosstalk channel gains within the group $g$. Furthermore, we denote the $n$-th user in group $g$ by $(g, n)$. The power spectral density (PSD) of user $(g, n)$ on tone $k$ before preconditioning is defined as $p_{k}^{(g, n)} \triangleq$ $\mathbb{E}\left\{\left|x_{k}^{(g, n)}\right|^{2}\right\} / \Delta f$ while the noise power density of user $(g, n)$ on tone $k$ is defined as $\sigma_{k}^{(g, n)} \triangleq \mathbb{E}\left\{\left|z_{k}^{(g, n)}\right|^{2}\right\} / \Delta f$, where $\mathbb{E}\{\cdot\}$ denotes the expectation operator. We assume that the channel matrix $\mathbf{H}_{k}^{g, g}$ is perfectly known and can be used for the calculation of the per-group crosstalk pre-compensation matrix. It has been shown in [13] that the diagonalizing precoder (DP) [14] also for G.fast achieves near-optimal bit-rates under the assumption of perfect channel knowledge. Thus, we consider DP as crosstalk cancellation scheme. Note that in this work we assume downstream transmission. However, the presented results can be easily adapted to the upstream case as well. DP multiplies the transmitted signal vector by a precoding matrix
$\mathbf{S}$ as follows:

$$
\mathbf{y}_{k}^{g}=\mathbf{H}_{k}^{g, g} \mathbf{S}_{k}^{g} \mathbf{x}_{k}^{g}+\sum_{g^{\prime} \neq g} \mathbf{H}_{k}^{g, g^{\prime}} \mathbf{S}_{k}^{g^{\prime}} \mathbf{x}_{k}^{g^{\prime}}+\mathbf{z}_{k}^{g},
$$

where $\mathbf{S}_{k}^{g}=\left(\beta_{k}^{g}\right)^{-1}\left(\mathbf{H}_{k}^{g, g}\right)^{-1} \operatorname{diag}\left\{\mathbf{H}_{k}^{g, g}\right\}$ with $\beta_{k}^{g}=$ $\max _{n \in \mathcal{N}_{g}}\left\|\left[\left(\mathbf{H}_{k}^{g, g}\right)^{-1}\right]_{\text {row } n} \operatorname{diag}\left\{\mathbf{H}_{k}^{g, g}\right\}\right\|_{2}$, which is a parameter selected to ensure compliance with the spectral mask constraint after the precoder [14], that is, $\beta_{k}^{g} \geq 1$. Note that $\operatorname{diag}\left\{\mathbf{H}_{k}^{g, g}\right\}$ is a diagonal matrix with the same diagonal elements as $\mathbf{H}_{k}^{g, g}$. Furthermore, we introduce the notation $\overline{\mathbf{H}}_{k}^{g, g}=\left(\beta_{k}^{g}\right)^{-1} \operatorname{diag}\left\{\mathbf{H}_{k}^{g, g}\right\}$ and $\overline{\mathbf{H}}_{k}^{g, g^{\prime}}=\mathbf{H}_{k}^{g, g^{\prime}} \mathbf{S}_{k}^{g^{\prime}}$. Based on Shannon's capacity formula the number of bits that can be reliably transmitted on a particular tone $k$ for user $(g, n)$ is determined by [11]

$$
b_{k}^{(g, n)}=\log _{2}\left(1+\frac{1}{\Gamma} \operatorname{SINR}_{k}^{(g, n)}\right),
$$

where $\operatorname{SINR}_{k}^{(g, n)}$ is the signal-to-interference-plus-noise ratio (SINR) of user $(g, n)$ on tone $k$, and $\Gamma$ denotes the SINR gap, which is a function of the desired bit error rate. The SINR of user $(g, n)$ on tone $k$ is given by

$$
\begin{aligned}
& \operatorname{SINR}_{k}^{(g, n)}=p_{k}^{(g, n)}\left|\bar{h}_{k}^{(g, n),(g, n)}\right|^{2} / \\
& \left(\sum_{m=1 ; m \neq n}^{N_{g}} p_{k}^{(g, m)}\left|\bar{h}_{k}^{(g, n),(g, m)}\right|^{2}+\right. \\
& \left.\sum_{g^{\prime}=1 ; g^{\prime} \neq g}^{G} \sum_{n^{\prime}=1}^{N_{g^{\prime}}} p_{k}^{\left(g^{\prime}, n^{\prime}\right)}\left|\bar{h}_{k}^{(g, n),\left(g^{\prime}, n^{\prime}\right)}\right|^{2}+\sigma_{k}^{(g, n)}\right),
\end{aligned}
$$

where $\bar{h}_{k}^{(g, n),\left(g^{\prime}, n^{\prime}\right)}$ denotes the crosstalk channel gain from user $\left(g^{\prime}, n^{\prime}\right)$ to user $(g, n)$ on tone $k$. Note that the first sum in the denominator corresponds to the crosstalk noise generated within one vectoring group and is entirely cancelled by DP (assuming perfect channel knowledge) while the second summation corresponds to the crosstalk noise generated among different groups and is avoided by means of DSM-L2 or IA, as we will discuss in the following section.

The bit-rate in bits-per-second (bps) for user $(g, n)$ is obtained by

$$
R^{(g, n)}=f_{s} \sum_{k}^{K} b_{k}^{(g, n)}
$$

where $f_{s}$ denotes the DMT symbol rate.

\section{INTERFERENCE MITIGATION FOR PARTIAL VECTORING}

\section{A. Joint vectoring and DSM-L2}

We assume that different vectoring groups are coordinated by DSM-L2. Shortly, DSM-L2 can be directly applied in the case of partial vectoring in order to avoid crosstalk noise between groups. More precisely, DSM-L2 is applied on top of vectoring and considers modified channel gains $\overline{\mathbf{H}}$ instead of the original ones in $\mathbf{H}$. 
The "traditional" objective in DSM is a sum-bit-rate maximization. This is sometimes also referred to as rate-adaptive DSM (RA-DSM). A typical RA-DSM design problem modified for partial vectoring case is formulated as follows:

$$
\begin{aligned}
& \underset{\left\{\mathbf{p}^{(g, n)}, \forall n \in \mathcal{N}_{g}, g \in \mathcal{G}\right\}}{\operatorname{maximize}} \sum_{g=1}^{G} \sum_{n=1}^{N_{g}} R^{(g, n)}, \\
& \text { s.t. } \sum_{k \in \mathcal{K}} \alpha_{k}^{(g, n)} p_{k}^{(g, n)} \leq P_{\text {tot }}^{(g, n)}, \forall n \in \mathcal{N}_{g}, g \in \mathcal{G}, \\
& \quad 0 \leq \alpha_{k}^{(g, n)} p_{k}^{(g, n)} \leq p_{k, \text { mask }}^{(g, n)}, \forall n \in \mathcal{N}_{g}, g \in \mathcal{G}, k \in \mathcal{K},
\end{aligned}
$$

where $\alpha_{k}^{(g, n)}=\left\|\left[\mathbf{S}_{k}^{g}\right]_{\text {row } n}\right\|_{2}^{2}, P_{\text {tot }}^{(g, n)}$ is the total transmit power budget for user $(g, n)$, and $p_{k, \text { mask }}^{(g, n)}$ is the PSD mask for user $(g, n)$ on tone $k$ at the output of the precoder. In this work we apply Iterative Spectrum Balancing (ISB) [3] to the problem in (7).

\section{B. Joint vectoring and IA}

Contrary to vectoring, interference alignment does not require all line termination units to be co-located and, therefore, is considered as alternative technique for crosstalk mitigation between groups. IA is a novel approach for dealing with interference in wireless communications where users experience strong interference and low SINR. Similarly, the strength of crosstalk channels in G.fast becomes comparable with that of the direct channel or even stronger especially on higher frequencies. Thus, G.fast interference channels resemble wireless interference channels in this respect and this motivates us to investigate application of IA in G.fast. IA cooperatively aligns interfering signals over time, space, or frequency dimensions which are usually defined as degrees of freedom (DoF). The IA problem is to design the decoders and precoders in such a way that the interfering signals at each receiver fall into a reduced-dimensional subspace. The receivers can then extract the projection of the desired signal that lies in the interferencefree subspace. When applied in a partial vectoring scenario, the aim of IA is to align the interference generated between groups. Thus, the original IA problem for user $n \in \mathcal{N}_{g}$ can be summarized as follows [9]

$$
\begin{gathered}
\left(\mathbf{U}^{(g, n)}\right)^{\dagger} \overline{\mathbf{H}}^{(g, n),\left(g^{\prime}, n^{\prime}\right)} \mathbf{V}^{\left(g^{\prime}, n^{\prime}\right)}=\mathbf{0}_{d^{(g, n)} \times d^{(g, n)}}, \forall g^{\prime} \neq g, \\
\operatorname{rank}\left(\left(\mathbf{U}^{(g, n)}\right)^{\dagger} \overline{\mathbf{H}}^{(g, n),(g, n)} \mathbf{V}^{(g, n)}\right)=d^{(g, n)},
\end{gathered}
$$

where $\overline{\mathbf{H}}^{(g, n),\left(g^{\prime}, n^{\prime}\right)} \in \mathbb{C}^{K \times K}$ contains crosstalk channel gains from user $\left(g^{\prime}, n^{\prime}\right)$ to user $(g, n), \mathbf{V}^{(g, n)}$ and $\mathbf{U}^{(g, n)}$ are $K \times d^{(g, n)}$ precoder and decoder matrices for user $(g, n)$, respectivley, and $d^{(g, n)}$ denotes the desired number of DoF for user $(g, n)$. For partial vectoring, IA precoder and decoder matrices are used to transform already modified channel gains $\overline{\mathbf{H}}$. Hence, the received signal of user $(g, n)$ after interference alignment is given by:

$$
\begin{aligned}
\widetilde{\mathbf{y}}^{(g, n)}=\left(\mathbf{U}^{(g, n)}\right)^{\dagger} \overline{\mathbf{H}}^{(g, n),(g, n)} \mathbf{V}^{(g, n)} \mathbf{x}^{(g, n)}+ \\
\sum_{g^{\prime}=1 ; g^{\prime} \neq g}^{G} \sum_{n^{\prime}=1}^{N_{g^{\prime}}}\left(\mathbf{U}^{(g, n)}\right)^{\dagger} \overline{\mathbf{H}}^{(g, n),\left(g^{\prime}, n^{\prime}\right)} \mathbf{V}^{\left(g^{\prime}, n^{\prime}\right)} \mathbf{X}^{\left(g^{\prime}, n^{\prime}\right)}+\widetilde{\mathbf{z}}^{(g, n)},
\end{aligned}
$$

where $\widetilde{\mathbf{y}}^{(g, n)}=\left(\mathbf{U}^{(g, n)}\right)^{\dagger} \mathbf{y}^{(g, n)}$ and $\widetilde{\mathbf{z}}^{(g, n)}=\left(\mathbf{U}^{(g, n)}\right)^{\dagger} \mathbf{z}^{(g, n)}$. Note that the summation corresponds to the crosstalk noise generated between groups and it is mitigated by the IA precoding and decoding matrices.

In this work we assume that users are connected with only one twisted-pair to the network premises. For this case, the DoF lie in frequency dimension due to the frequency selectivity of the DSL channel. Note that if DSL users were connected with more than one twisted pair to the network premises we could also consider interference alignment in the space dimension, i.e., over multiple-input multiple-output (MIMO) channels. However, in this work we consider the problem of interference alignment over single-input singleoutput (SISO) frequency selective channels. When IA is done in the frequency dimension, the complexity of IA grows linearly with the number of users and quadratically with the number of tones, i.e., $\mathcal{O}\left(N K^{2}\right)$ [10]. However, the scalability of interference alignment can significantly reduce this complexity as we will discuss later (cf. Section IV).

There are many algorithms in the literature for finding IA precoding and decoding matrices. In general, IA algorithms can be divided into two groups: a) algorithms which seek perfect interference alignment (constraint (8) is fulfilled) and b) algorithms which allow some amount of interference in the signal subspace but tend to maximize some other metrics (e.g., SINR or sum rate). It has been shown that for low to moderate SINR the latter ones yield higher bit rates [15], [16]. Therefore, in this work we use the Max-SINR algorithm (Algorithm 2 in [15]). Note that authors in [10] use the IA algorithm from the first group of IA algorithms (Algorithm 1 from [15]) in their evaluation and potentially do not gain full benefits from IA for moderate and low SINR scenarios. The Max-SINR iterates over users and updates matrices $\mathbf{V}^{(g, n)}$ and $\mathbf{U}^{(g, n)}$ to maximize the SINR. However, there is no proof that Max-SINR converges.

The objective function of each user $(g, n)$ is given by

$$
S I N R^{(g, n)}=\frac{\overline{\overline{\mathbf{H}}}^{(g, n),(g, n)} \mathbf{T}^{(g, n)}\left(\overline{\overline{\mathbf{H}}}^{(g, n),(g, n)}\right)^{\dagger}}{\mathbf{Q}^{(g, n)}+\mathbf{C}^{(g, n)}},
$$

where $\overline{\mathbf{H}}^{(g, n),(g, n)}=\left(\mathbf{U}^{(g, n)}\right)^{\dagger} \overline{\mathbf{H}}^{(g, n),(g, n)} \mathbf{V}^{(g, n)}, \mathbf{C}^{(g, n)}=$ $\sum_{g^{\prime}=1 ; g^{\prime} \neq g}^{G} \sum_{n^{\prime}=1}^{N_{g^{\prime}}} \overline{\overline{\mathbf{H}}}^{(g, n),\left(g^{\prime}, n^{\prime}\right)} \mathbf{T}^{\left(g^{\prime}, n^{\prime}\right)}\left(\overline{\overline{\mathbf{H}}}^{(g, n),\left(g^{\prime}, n^{\prime}\right)}\right)^{\dagger}$ is the interference covariance matrix where $\overline{\overline{\mathbf{H}}}^{(g, n),\left(g^{\prime}, n^{\prime}\right)}=\left(\mathbf{U}^{(g, n)}\right)^{\dagger} \overline{\mathbf{H}}^{(g, n),\left(g^{\prime}, n^{\prime}\right)} \mathbf{V}^{\left(g^{\prime}, n^{\prime}\right)}$ and $\mathbf{Q}^{(g, n)}=$ $\sigma^{(g, n)}\left(\mathbf{U}^{(g, n)}\right)^{\dagger}\left(\mathbf{U}^{(g, n)}\right)$ is the noise covariance matrix of user $(g, n)$. Matrix $\mathbf{T}^{(g, n)}=\operatorname{diag}\left\{p_{1}^{(g, n)}, p_{2}^{(g, n)}, \ldots, p_{K}^{(g, n)}\right\}$ contains the PSD of user $(g, n)$ over all $K$ tones. We assume that all $N$ users are perfectly synchronized, i.e., $\mathbf{H}^{(g, n),(g, n)}=\operatorname{diag}\left\{\bar{h}_{1}^{(g, n),(g, n)}, \ldots, \bar{h}_{K}^{(g, n),(g, n)}\right\}$. 
Each user $(g, n)$ solves the optimization problem

$$
\begin{array}{r}
\underset{\left\{\mathbf{V}^{(g, n)}, \mathbf{U}^{(g, n)}, \forall g \in \mathcal{G}, n \in \mathcal{N}_{g}\right\}}{\operatorname{maximize}} \operatorname{SIN} R^{(g, n)} \\
\text { s.t. }\left\|V^{(g, n)}\right\|_{2}^{2}=1, \forall n \in \mathcal{N}_{g}, g \in \mathcal{G} \\
\left\|U^{(g, n)}\right\|_{2}^{2}=1, \forall n \in \mathcal{N}_{g}, g \in \mathcal{G}
\end{array}
$$

where (12b) and (12c) are subject to the transmit power constraints and $\|.\|_{2}^{2}$ denotes the squared $L 2$ norm.

\section{INTERFERENCE ALIGNMENT IMPLEMENTATION ISSUES}

Taking into consideration that G.fast systems will use up to 4096 tones, we see how this imposes high computational complexity on IA algorithms. The scalability of interference alignment can significantly reduce this complexity as proposed in [10]. The main idea is to divide the whole available spectrum into $K_{s}$ sub-bands with $\triangle K_{s}$ tones pre sub-band where the interference alignment is done per each sub-band instead over all tones. Hence, IA complexity depends on $\triangle K_{s}$ instead of $K$, i.e., $\mathcal{O}\left(N K_{s} \triangle K_{s}^{2}\right)$.

Another important factor with respect to the implementation of IA is the diversity of the DSL channel. For SISO channels, diversity both in space and frequency dimensions play an important role in making IA feasible. Under space diversity we assume different crosstalk channels between different users i.e., $\bar{h}_{k}^{(g, n),\left(g^{\prime}, n^{\prime}\right)} \neq \bar{h}_{k}^{(g, n),\left(g^{\prime}, n^{\prime \prime}\right)}$ while frequency diversity is essentially the frequency selectivity of the channel i.e., $\bar{h}_{k}^{(g, n),\left(g^{\prime}, n^{\prime}\right)} \neq \bar{h}_{l}^{(g, n),\left(g^{\prime}, n^{\prime}\right)}$. Based on measurement results [7], [8] we see that DSL exhibits a frequency-selective crosstalk with variations in crosstalk power from one twisted pair to another. Thus, both frequency and spatial diversity requirements are fulfilled. However, the frequently used $99 \%$ worst-case model [11] does not capture entirely the natural diversity of DSL channels. Note that authors in [10] use $99 \%$ worst-case crosstalk model and thus potentially obtain lower bit-rates due to the "poor" diversity of simulated crosstalk channels. In this work we consider crosstalk model proposed in [12] where crosstalk dispersion feature is added in order to overcome the limitations of $99 \%$ worst-case model. The crosstalk model is defined as [12]

$$
H_{\mathrm{Ex}-F E X T}(f)=\left|H_{F E X T}(f, d)\right| e^{j \varphi(f)} 10^{-0.05 X(f)},
$$

where $\left|H_{F E X T}(f, d)\right|$ is the crosstalk amplitude obtained with $99 \%$ wost-case model. $\varphi(f)$ and $X(f)$ are random variables modelling the channel phase and amplitude dispersion, respectively.

\section{Simulation Results}

In this section, we consider two scenarios using the network topology depicted in Fig. 1:

a) Vectoring is not feasible. Crosstalk noise between users is mitigated with ISB, IA, or TDMA.

b) Vectoring is feasible for users within two or three vectoring groups. For two vectoring group case, users $\{1,2,3,7,8,9,13,14,15,19,20,21\}$ belong to the first group while all others belong to the second group.

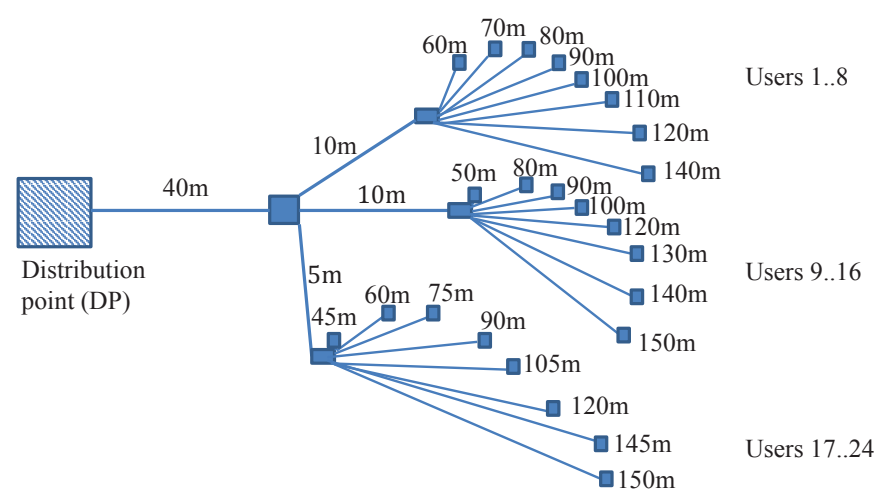

Fig. 1. Distributed DSL access network.

For the case with three vectoring groups, users $\{1,4,7,10,13,16,19,22\},\{2,5,8,11,14,17,20,23\}$, and $\{3,6,9,12,15,18,21,24\}$ belong to the first, second, and third group. Crosstalk noise among groups is mitigated by ISB, IA or TDMA. As a reference we also consider the case where no interference mitigation between groups is applied.

We evaluate performance for two frequency bands: 2.2 MHz-106 MHz and $106 \mathrm{MHz}-212 \mathrm{MHz}$. We consider different frequency bands because the G.fast standard recommends different DSM techniques for frequencies up to $106 \mathrm{MHz}$ and $212 \mathrm{MHz}$. Thus, we show the results separately in order to give clear guidelines for two G.fast frequency bands. We consider the TP100 cable model [17] and frequency flat background noise equal to $-140 \mathrm{dBm} / \mathrm{Hz}$. All users transmit with $-76 \mathrm{dBm} / \mathrm{Hz}$ and with DMT symbol rate $f_{s}=48 \mathrm{kHz}$. The tone spacing $\Delta f$ and SINR gap are set to $51.75 \mathrm{kHz}$ and $10.75 \mathrm{~dB}$, respectively. As already mentioned, we use crosstalk model proposed in [12] with dispersion parameters $X(f)=3 \mathrm{~dB}$ and $\varphi(f)=0 \mathrm{~dB}$ (cf. Eq. (13)). Furthermore, interference alignment is achieved by independently aligning the interference on $K_{s}$ sub-bands where number of iterations of the Max-SINR algorithm per each sub-band is 250. We assume $\triangle K_{s}>1$ and $K_{s}=\left\lceil K / \triangle K_{s}\right\rceil$, where the last sub-band may have less than $\triangle K_{s}$ tones.

\section{A. Results for frequency band $2.2 \mathrm{MHz}-106 \mathrm{MHz}$}

Table I shows the sum rates for different numbers of vectoring groups and for different interference mitigation techniques. Results are compared to network capacity (i.e. interference free scenario) where achievable sum rates are limited only by background noise. Results show that in case when vectoring is feasible over all users (one vectoring group) we are able to achieve $98 \%$ of network capacity. Furthermore, we notice that in scenario where vectoring is not feasible IA achieves the highest sum rate and it is outperforming ISB and TDMA by $11.5 \%$ and $222 \%$, respectively. For comparison we also evaluate IA and ISB performance on lower frequencies $(\leq 30 \mathrm{MHz})$ where currently deployed DSL systems are operating, e.g. Very High Speed Digital Subscriber Line 2 (VDSL2). In our simulation we consider three VDSL2 band plans: B7-1, B8-4, 


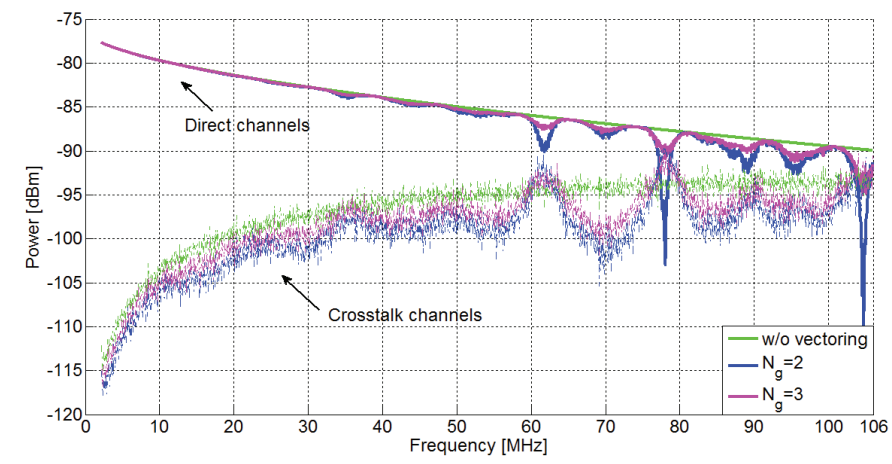

Fig. 2. The received power of direct channels and crosstalk channels in $2.2 \mathrm{MHz}$ to $106 \mathrm{MHz}$ band for user 1 (cf. Fig. 1) and different number of vectoring groups $\left(N_{g}\right)$.

and B8-15 [18] which use frequencies up to $8 \mathrm{MHz}, 17 \mathrm{MHz}$, and $30 \mathrm{MHz}$, respectively. Simulation results reveal that in lower frequencies where the SINR is still considerably high (cf. Fig. 2) ISB performs better than IA achieving 64\%, $58 \%$, and $30 \%$ higher sum rates for B7-1, B8-4, and B8-15 band plans, respectively.

For partial vectoring, we notice that the effect of crosstalk originating from different vectoring groups is in large extent mitigated by interference mitigation techniques. We also notice that there is a substantial drop in achievable sum rates when number of vectoring groups increases from two to three. More precisely, sum rates drop from $50 \%$ to $30 \%$ depending on the interference mitigation scheme applied. Thus, for partial vectoring in G.fast, the number of vectoring groups should be kept as small as possible. Furthermore, the results from Table I show that sum rates under joint vectoring/ISB and joined vectoring/IA are equal or even (slightly) lower than those under simple joint vectoring/TDMA implementation. This is somehow contrary to conclusions made for previous DSL systems where joint vectoring/ISB (or its alternatives) was shown to be optimal solution for partial vectoring [5]. For comparison we also evaluate performance of joint vectoring/ISB for three VDSL2 band plans: B7-1, B8-4, and B8-15 [18]. Simulation results show that joint vectoring/ISB achieves $58 \%, 54 \%$, and $52 \%$ of network capacity for two groups and $55 \%, 42 \%$, and $33 \%$ of capacity for three groups and VDSL2 band plans B7-1, B8-4, and B8-15, respectively. Thus, we see that in lower frequencies $(\leq 30 \mathrm{MHz})$ joint vectoring/ISB performs better or eventually equal as TDMA. This can be explained as follows. In higher frequencies the noise power received over crosstalk channels becomes stronger while the power received over direct channel decreases due to the stronger attenuation at higher frequencies, which results in lower SINR in higher frequencies, cf. Fig 2. For low SINR optimal solution for interference mitigation starts to resemble a simple solution where each group uses one portion of the available bandwidth. In fact, this is exactly the working principle of TDMA.

\section{B. Results for frequency band $106 \mathrm{MHz}-212 \mathrm{MHz}$}

In Table II we show simulation results for second frequency band, i.e., $106 \mathrm{MHz}-212 \mathrm{MHz}$ band. Results indicate that when

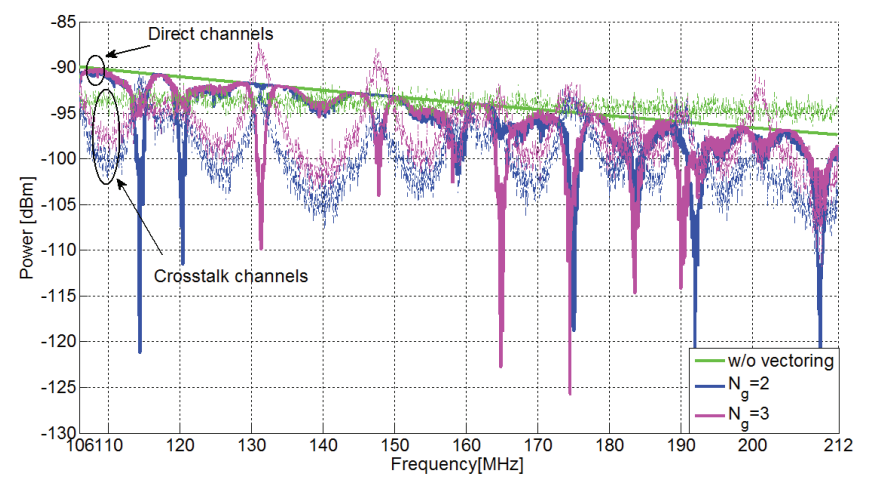

Fig. 3. The received power of direct channels and crosstalk channels in $106 \mathrm{MHz}-212 \mathrm{MHz}$ band for user 1 (cf. Fig. 1) and different number of vectoring groups $\left(N_{g}\right)$.

TABLE I

SiMULATION RESULTS FOR THE $2.2 \mathrm{MHz}-106 \mathrm{MHz}$ BAND.

\begin{tabular}{|l|l|l|l|l|}
\hline Vectoring & $\begin{array}{l}\text { Interference } \\
\text { mitigation }\end{array}$ & $N_{g}$ & $\begin{array}{l}\text { Sum rate } \\
{[\mathrm{Mbps}]}\end{array}$ & $\begin{array}{c}\text { \% of } \\
\text { network } \\
\text { capacity }\end{array}$ \\
\hline $\mathrm{N}$ & - & - & 2391.8 & 9.3 \\
\hline $\mathrm{N}$ & TDMA & - & 1064.8 & 4.2 \\
\hline $\mathrm{N}$ & ISB & - & 3077.9 & 12 \\
\hline $\mathrm{N}$ & $\mathrm{IA}$ & - & 3433.8 & 13.5 \\
\hline $\mathrm{Y}$ & - & 1 & 25013.8 & 98 \\
\hline $\mathrm{Y}$ & - & 2 & 3524.9 & 13.8 \\
\hline $\mathrm{Y}$ & TDMA & 2 & 12777.7 & 50 \\
\hline $\mathrm{Y}$ & ISB & 2 & 12763.9 & 50 \\
\hline $\mathrm{Y}$ & IA & 2 & 12545.6 & 49 \\
\hline $\mathrm{Y}$ & - & 3 & 3005.2 & 11.8 \\
\hline $\mathrm{Y}$ & TDMA & 3 & 8518.4 & 33 \\
\hline $\mathrm{Y}$ & ISB & 3 & 8330.2 & 32.6 \\
\hline $\mathrm{Y}$ & IA & 3 & 6744.5 & 26.4 \\
\hline
\end{tabular}

vectoring is feasible over all users we are able to achieve $93 \%$ of the network capacity. For the $2.2 \mathrm{MHz}-106 \mathrm{MHz}$ band (cf. Section V-A) vectoring achieved $98 \%$ of network capacity. Higher bit-rate loss in $106 \mathrm{MHz}-212 \mathrm{MHz}$ band is explicable by the higher transmit power scaling $\left(\beta_{k}\right)$ on higher frequencies due to the stronger crosstalk noise as shown in [19]. Furthermore, when vectoring is not feasible IA again achieves the highest sum rates. More precisely, for this frequency band IA achieves $95 \%$ and $202 \%$ higher sum rates compared to ISB and TDMA, respectively. This should be compared with results obtained for the $2.2 \mathrm{MHz}-106 \mathrm{MHz}$ band where IA achieved $11.5 \%$ higher sum rate compared to ISB. From Fig. 3 we see how power received over crosstalk channels becomes dominant with respect to the power received over the direct channel for frequencies above $106 \mathrm{MHz}$. This results in lower SINR received in $106 \mathrm{MHz}-212 \mathrm{MHz}$ band compared to $2.2 \mathrm{MHz}-106 \mathrm{MHz}$ band. Combining this with the results from Section V-A we conclude that IA benefits from strong interference unlike ISB and, therefore, becomes more efficient interference mitigation technique than ISB (and TDMA) for G.fast frequencies, especially for $106 \mathrm{MHz}-212 \mathrm{MHz}$ band.

For partial vectoring and without interference mitigation mechanisms applied, achievable sum rates drop compared 
TABLE II

SIMULATION RESULTS FOR THE 106 MHz-212 MHz BAND.

\begin{tabular}{|l|l|l|l|l|}
\hline Vectoring & $\begin{array}{l}\text { Interference } \\
\text { mitigation }\end{array}$ & $N_{g}$ & $\begin{array}{l}\text { Sum rate } \\
{[\mathrm{Mbps}]}\end{array}$ & $\begin{array}{c}\text { \% of } \\
\text { network } \\
\text { capacity }\end{array}$ \\
\hline $\mathrm{N}$ & - & - & 0 & 0 \\
\hline $\mathrm{N}$ & TDMA & - & 714 & 4.2 \\
\hline $\mathrm{N}$ & ISB & - & 1103.4 & 6.4 \\
\hline $\mathrm{N}$ & $\mathrm{IA}$ & - & 2156.3 & 12.6 \\
\hline $\mathrm{Y}$ & - & 1 & 15927.2 & 93 \\
\hline $\mathrm{Y}$ & - & 2 & 65.6 & 0.4 \\
\hline $\mathrm{Y}$ & TDMA & 2 & 8568.4 & 50 \\
\hline $\mathrm{Y}$ & ISB & 2 & 8085.4 & 47.2 \\
\hline $\mathrm{Y}$ & IA & 2 & 7828.8 & 45.7 \\
\hline $\mathrm{Y}$ & - & 3 & 0.2 & 0 \\
\hline $\mathrm{Y}$ & TDMA & 3 & 5712.2 & 33 \\
\hline $\mathrm{Y}$ & ISB & 3 & 5128.5 & 30 \\
\hline $\mathrm{Y}$ & IA & 3 & 5517.1 & 32.2 \\
\hline
\end{tabular}

to the $2.2 \mathrm{MHz}-106 \mathrm{MHz}$ band approaching approximately $0 \mathrm{Mbps}$ for three vectoring groups. ISB and IA improve the achievable sum rate in case of partial vectoring. However, the best results are again achieved using jointly vectoring and TDMA. Except achieving the highest sum rate, TDMA is also less complex compared to ISB and IA. Furthermore, it is inherently present in G.fast systems and, therefore, does not require any additional implementation changes unlike IA.

To summarize, the results indicate that IA achieves better performance compared to ISB and TDMA in case when vectoring is not feasible. From presented results we can also conclude that as crosstalk noise power increases IA bit rate gains over ISB and TDMA increase as well. For partial vectoring, the joint vectoring/TDMA approach yields the highest sum rates compared to considered interference mitigation techniques.

\section{CONCLusions}

G.fast is the next generation digital subscriber line technology recently standardized within the International Telecommunication Union. Due to strong interference which becomes dominant on high frequencies (e.g., above $100 \mathrm{MHz}$ ), Gigabit bit-rates can only be achieved by using vectoring. We considered diagonalizing precoding (DP) and showed that it is capable of achieving $98 \%$ of the interference-free capacity on frequencies below $106 \mathrm{MHz}$. On frequencies between $106 \mathrm{MHz}$ and $212 \mathrm{MHz}$ DP suffers from higher bitrate losses due to power normalization but still reaches $93 \%$ of the interference-free capacity. However, vectoring requires coordination of signals at the line termination side. In this work, we also studied the bit-rates achievable by G.fast when vectoring is not feasible or only applied within groups of users (partial vectoring). For the mitigation of crosstalk among groups we considered iterative spectrum balancing (ISB), interference alignment (IA), and time division multiple access (TDMA). Simulation results indicate that in the case when vectoring is not feasible, IA achieves higher sum rates than ISB and TDMA. For partial vectoring, the results show that the achievable bit-rates drop substantially as the number of vectoring groups increases. Thus, for partial vectoring in G.fast the number of groups should be kept as small as possible. Furthermore, our results indicate that in case of partial vectoring TDMA achieves the best results among the considered crosstalk mitigation techniques.

\section{REFERENCES}

[1] "Updated draft text for G.fast - version 11.0," ITU-T SG15/Q4a Contribution,2013-12-Q4-R20R6, Geneva, Switzerland, December 2013.

[2] W. Yu, G. Ginis, and J. Cioffi, "Distributed multiuser power control for digital subscriber lines," IEEE Journal on Selected Areas in Communications, vol. 20, no. 5, pp. 1105-1115, June 2002.

[3] R. Cendrillon and M. Moonen, "Iterative spectrum balancing for digital subscriber lines," IEEE International Conference on Communications (ICC), vol. 3, pp. 1937-1941, May 2005.

[4] G. Ginis and J. Cioffi, "Vectored transmission for digital subscriber line systems," IEEE Journal on Selected Areas in Communications, vol. 20, no. 5, pp. 1085-1104, June 2002.

[5] A. Forouzan, M. Moonen, J. Maes, and M. Guenach, "Joint level 2 and 3 dynamic spectrum management for downstream DSL," Communications, IEEE Transactions on, vol. 60, no. 10, pp. 3111-3122, October 2012.

[6] _ "Joint level 2 and 3 dynamic spectrum management for upstream VDSL," Communications, IEEE Transactions on, vol. 59, no. 10, pp. 2851-2861, October 2011.

[7] M. Timmers, M. Guenach, C. Nuzman, and J. Maes, "G.fast: evolving the copper access network," IEEE Communications Magazine, vol. 51, no. 8, pp. 74-79, August 2013.

[8] P. T. B. van den Heuvel and R. van den Brink, "G.fast: Preliminary analysis of the transfer characteristics of the $104 \mathrm{~m} \mathrm{KPN}$ access cable," ITU-T SG15/Q4a, Contribution 2013-03-Q4-026, Red Bank, New Jersey, March 2013.

[9] V. Cadambe and S. Jafar, "Interference alignment and degrees of freedom of the K-user interference channel," IEEE Transactions on Information Theory, vol. 54, no. 8, pp. 3425-3441, August 2008

[10] S. Huberman and T. Le-Ngoc, "Interference alignment for DSL," Global Communications Conference (GLOBECOM), pp. 3092-3097, December 2012.

[11] P. Golden, H. Dedieu, and K. Jacobsen, Eds., Fundamentals of DSL technology. Auerbach Publications, 2006.

[12] M. Sorbara, P. Duvaut, F. Shmulyian, S. Singh, and A. Mahadevan, "Construction of a DSL-MIMO channel model for evaluation of FEXT cancellation systems in VDSL2," IEEE Sarnoff Symposium, pp. 1-6, April 2007.

[13] S. Drakulić, D. Statovci, and M. Wolkerstorfer, "Performance of linear crosstalk cancelation in fourth generation wired broadband access networks," Proceedings of the 21st European Signal Processing Conference (EUSIPCO), pp. 1-5, September 2013.

[14] R. Cendrillon, G. Ginis, E. Van den Bogaert, and M. Moonen, "A NearOptimal Linear Crosstalk Precoder for Downstream VDSL," in IEEE Transactions on Communications, vol. 55, no. 5, pp. 860-863, May 2007.

[15] K. Gomadam, V. Cadambe, and S. Jafar, "Approaching the capacity of wireless networks through distributed interference alignment," IEEE Global Telecommunications Conference (GLOBECOM), pp. 1-6, November 2008.

[16] R. Brandt, H. Asplund, and M. Bengtsson, "Interference alignment in frequency - a measurement based performance analysis," International Conference on Systems, Signals and Image Processing (IWSSIP), pp. 227-230, April 2012.

[17] R. F. M. van den Brink, "Cable reference models for simulating metallic access networks," ETSI STC TM6 Permanent document, TM6(97)02, Tech. Rep., June 1998.

[18] ITU, Very high speed digital subscriber line transceivers 2 (VDSL2), Technical Recommendation G.993.2, ITU Std., July 2002.

[19] P. E. Eriksson, C. Lu, and I. Pappa, "Performance study on using linear precoding (vectoring) for high frequency DSL," ITU-T SG15/Q4a Contribution COM15-C 1489-E, February 2011. 